\title{
Defect indications in sono-thermography in relation to defect location and structure
}

\author{
by G. Walle, M. Abuhamad, E. Toma, and U. Netzelmann, \\ Fraunhofer Institute for Nondestructive Testing, Saarbrücken, Germany
}

\begin{abstract}
A high power ultrasonic burst excitation technique was used to study the potential of the ultrasound excited thermography (vibro- or sono-thermography) to detect and to estimate the depth position of cracks in ceramics as well as voids in glass fibre reinforced polymer. By verification using high-frequency $(50 \mathrm{MHz})$ ultrasound techniques it was proven that sono-thermography can detect relatively deep defects in ceramics. The depth of the defect can be estimated using a theoretical model based on analytical calculations dealing with friction heat sources equally distributed over the crack flanks stimulated by the ultrasonic vibrations of the specimen. In carbon fibre reinforced polymers, the thermographic indications of defects were correlated with 3D X-ray cross sections of the specimen.
\end{abstract}

\section{Introduction}

High power ultrasound excited thermography or sono-thermography turned out to be successful for fast detection of cracks in various types of materials [1, 2]. Even on complex shaped components like turbine blades, cracks become visible in dark-field contrast infrared images within short inspection times. This suggests sonothermography to be used as a routine tool for $100 \%$ production quality testing.

In this paper we present the application of the technique to the characterisation of sub-surface cracks in ceramic material as well as in fibre reinforced polymer. The thermal signatures obtained in ultrasonic burst excited thermography are correlated with results of the defect position and structure obtained by other non-destructive techniques.

\section{Sample}

A fine-grained clay based ceramic specimen was containing an artificially provoked crack that was a trigger for generation of a larger natural crack during sintering. Another specimen examined in this study was a component of glass fibre reinforced polymer containing internal voids.

\section{Experimental}

The ceramic specimen was excited by an high intensity ultrasonic burst pulse with an ultrasound frequency of $20.5 \mathrm{kHz}$ and a pulse duration of $0.8 \mathrm{~s}$. As measurement system, a mid-wave infrared focal plane array (256 × 256 pixels) camera was used. The infrared camera renders a frame spacing of $5 \mathrm{~ms}$ (at highest pixel number) and a temperature resolution of about $30 \mathrm{mK}$. The measurement 


\section{http://dx.doi.org/10.21611/qirt.2004.033}

system is controlled via the VISOTHERM ${ }^{\circledR}$ software package. The software controls the ultrasonic heating via a sonotrode coupled onto the specimen surface using a pneumatic press. On the other hand the software overtakes the image acquisition in synchronisation to the ultrasonic heating as well as the processing, evaluation and presenting of the measurement results.

\section{Results}

In figure 1, a thermographic image of the ceramic specimen is presented. The image shows the temperature distribution about $4 \mathrm{~s}$ after the begin of ultrasonic heating $(20.5 \mathrm{kHz}, 0.8 \mathrm{~s})$ using a sonotrode on the top surface as well as on the front surface of the specimen (inclination angle of view: about $45^{\circ}$ ). On the left side one can see the heating spot due to the ultrasonic excitation on the surface. The thermal response of the crack tips is visible at the outer specimen front surface (at this time already blurred). In the centre of the top surface a further temperature contrast can be recognised. The time dependence is strongly different from the contrasts of the front surface breaking crack-tips and suggests a sub-surface defect source.

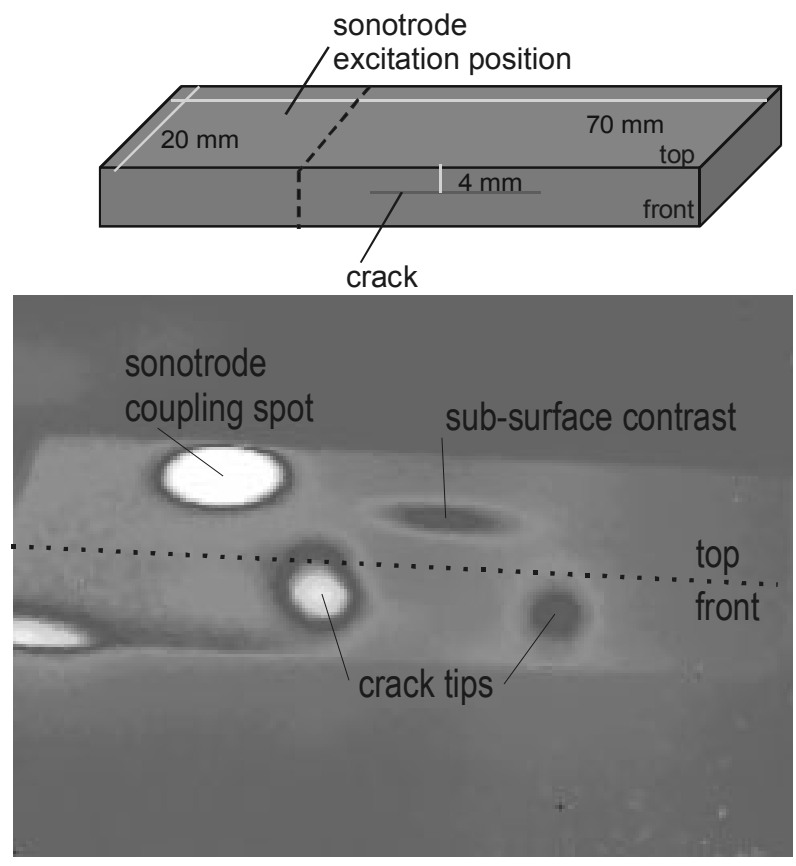

Fig. 1. Sketch of the ceramic specimen (top) and thermographic image of the temperature distribution about $4 s$ after begin of heating (bottom) 


\section{http://dx.doi.org/10.21611/qirt.2004.033}

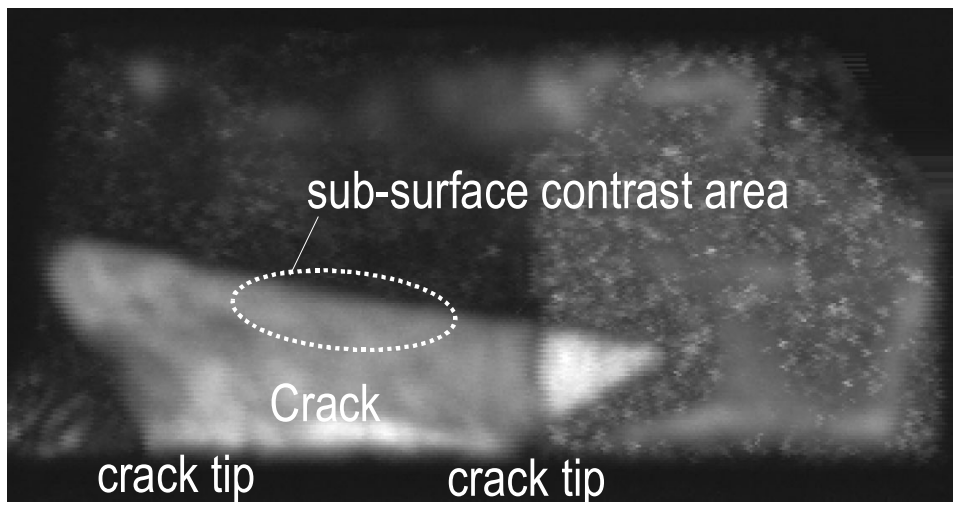

Fig. 2. High-frequency ultrasound (50 MHz) C-scan of the ceramic, top side view, revealing the extension of the internal crack (bright grey structure). The area of the sub-surface thermal contrast in figure 1 is indicated by the dashed curve (image size $50 \mathrm{~mm} \times 25 \mathrm{~mm}$ )

The thermographic results were correlated with high-resolution ultrasonic Cand B-scans of the specimen. These allow to image the shape and depth of the heat generating crack by independent, non-destructive means (figure 2). In the C-scan shown in figure 2, the bright contrast corresponds to high acoustic scattering and reflection from the inner volume of the ceramic. From B-scans, the defect depth can be determined by time-of-flight measurement and the independently measured speed of sound in the specimen. In this way the depth of the crack was determined to be about $2.5 \mathrm{~mm}$ below the surface in area of the sub-surface contrast in the thermographic image of figure 1.

\section{Theoretical considerations}

An analytical two-dimensional model [3] was chosen assuming an infinitely long parallelepiped with a width of $b$ and a height of $a$. Friction heat sources with $a$ power density $\mathrm{p}$ are equally distributed over a stripe area (width: $\Delta \mathrm{x}=\mathrm{x}_{2}-\mathrm{x}_{1}$ ) were considered (figure 3).

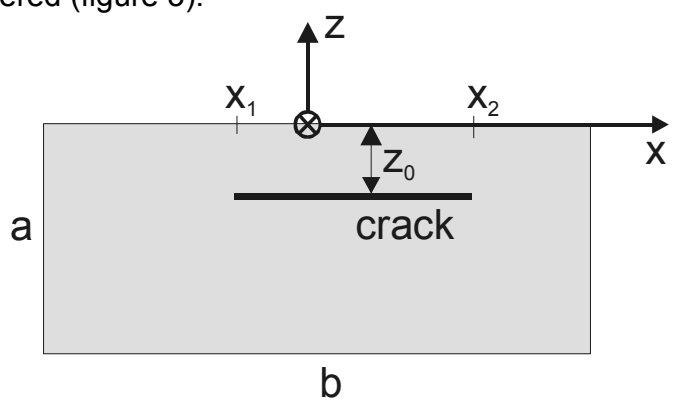

Fig. 3. Sketch of the model situation (cross section)

The crack is positioned in a plane parallel to the top surface of a parallelepiped with a distance $z_{0}$ from this surface. 
For the case that the heat sources (due to ultrasonic excitation) are active for the pulse duration $t_{p}$ the temperature at a point $x_{0}$ on the front surface $(z=0)$ is:

$$
\begin{aligned}
& T(0, t)=T_{1}+T_{2}+T_{3}, \text { where } \\
& T_{1}=\frac{p}{\rho \operatorname{cab}}\left(t_{p}\left(x_{2}-x_{1}\right)+\frac{2 a^{2}\left(x_{2}-x_{1}\right)}{\alpha \pi^{2}} \sum_{n=1}^{\infty} \frac{1}{n^{2}} e^{-n^{2} \beta t}\left(e^{n^{2} \beta t_{p}}-1\right) \cos \left(n \pi \frac{z_{0}}{a}\right)\right), \\
& T_{2}=\frac{p}{\rho c a b} \frac{2 b^{2}}{\alpha \pi^{2}} \sum_{m=1}^{\infty} \frac{1}{m^{2}} \frac{b}{m \pi} e^{-m^{2} \beta_{1} t}\left(e^{m^{2} \beta_{1} t_{p}}-1\right) \cos \left(m \pi \frac{x_{0}}{b}\right), \\
& \cdot\left(\sin \left(m \pi \frac{x_{2}}{b}\right)-\sin \left(m \pi \frac{x_{1}}{b}\right)\right) \\
& T_{3}=\frac{p}{\rho \operatorname{cob}} \frac{4 a^{2} b^{2}}{\alpha \pi^{2}} \sum_{n=1}^{\infty} \sum_{m=1}^{\infty} \frac{1}{n^{2} b^{2}} \frac{1}{m^{2} a^{2}} \frac{b}{m \pi} e^{-\beta_{2} t}\left(e^{\beta_{2} t_{p}}-1\right) \cos \left(n \pi \frac{z_{0}}{a}\right) \\
& \cdot \cos \left(m \pi \frac{x_{0}}{b}\right)\left(\sin \left(m \pi \frac{x_{2}}{b}\right)-\sin \left(m \pi \frac{x_{1}}{b}\right)\right)
\end{aligned}
$$

and

$$
\beta=\frac{\alpha \pi^{2}}{\mathrm{a}^{2}}, \quad \beta_{1}=\frac{\alpha \pi^{2}}{\mathrm{~b}^{2}}, \beta_{2}=\alpha \pi^{2}\left(\frac{1}{\mathrm{n}^{2} \mathrm{~b}^{2}}+\frac{1}{\mathrm{~m}^{2} \mathrm{a}^{2}}\right),
$$

$\rho:$ density,

c: specific heat capacity,

$\alpha$ : thermal diffusivity.

Using typical literature thermal material parameters of the studied ceramics the time dependence of temperature at the surface was calculated. The absolute surface thermal contrast and its time dependence could be brought into a relatively good match with the experimental results if the power density of the heat sources was set to $2.7 \mathrm{~W} / \mathrm{cm}^{2}$, the depth position of the heat sources $\left(z_{0}\right)$ was $2.5 \mathrm{~mm}$ (as measured by the ultrasound B-scans) and if the width $\Delta \mathrm{x}$ of the defect was set to 5 $\mathrm{mm}$. The pulse duration was chosen to $0.8 \mathrm{~s}$ as in the experiment. In figure 4 the calculated curve of the time dependence of the temperature contrast at the top surface of the ceramic material is presented (at $x_{0}=0, x_{1}=-2.5 \mathrm{~mm}, \mathrm{x}_{2}=2.5 \mathrm{~mm}$ ) in comparison with the measurement result at the centre of the sub-surface thermal contrast (see also figure 3). The discrepancy which can be found especially at later times can be interpreted by the fact that in the model it was assumed a defect producing a uniform distribution of heat sources over a stripe with an infinite extension in y-direction while in reality the heat sources are limited to the finite crack length. Furthermore, heat transfer by radiation or convection at the front surface as well as on the back surface of the specimen were not regarded in the model. 


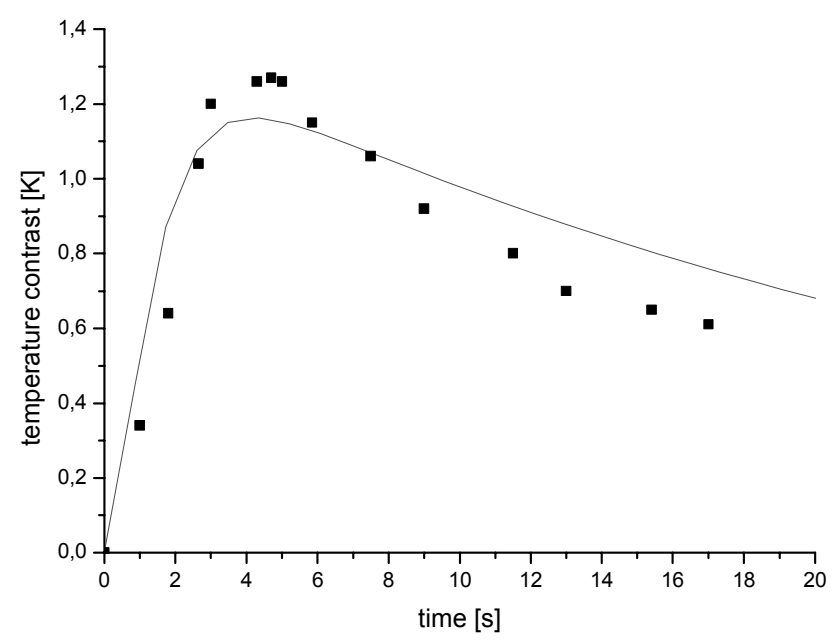

Fig. 4. Calculated time dependence (continuous curve) of the sub-surface defect thermal contrast in comparison to the measurement (black points)

Another comparison between sono-thermographic crack indications and independent non-destructive techniques was applied to structures made out of long glass fibre reinforced thermoplastic polymers. Here, 3D X-ray computed tomography served as a reference. Figure 5 shows, beside heating around the sonotrode coupling spot, an unexpected area with a strong thermal contrast. The X-ray tomography revealed internal voids and a porous structure (not visible from outside). The correlation between the position of thermal surface contrasts and internal defects was very good (figure 6).

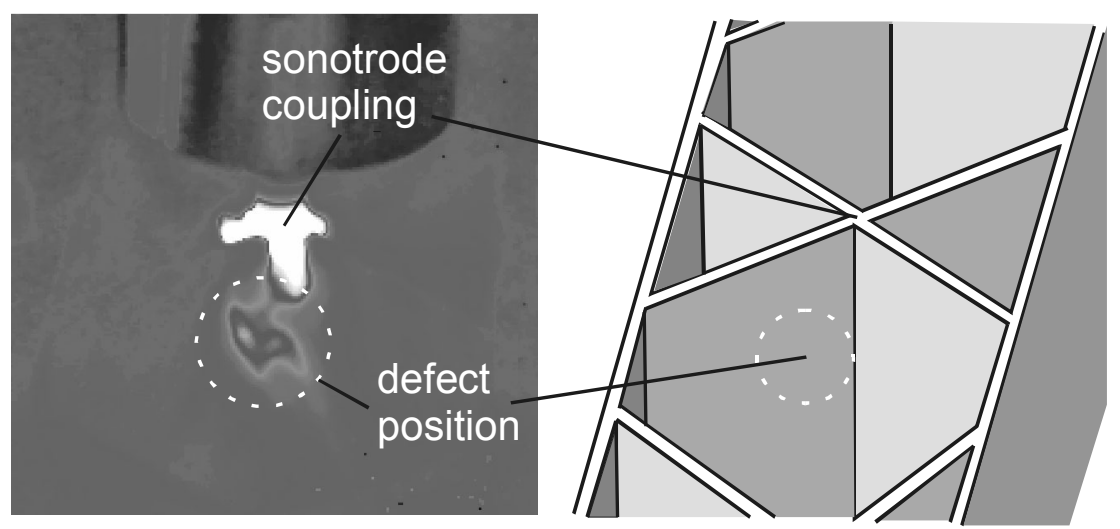

Fig. 5: Left: Thermographic image of a specimen with a sub-surface delamination; right side: sketch of the component as it appears in the IR camera view 


\section{http://dx.doi.org/10.21611/qirt.2004.033}

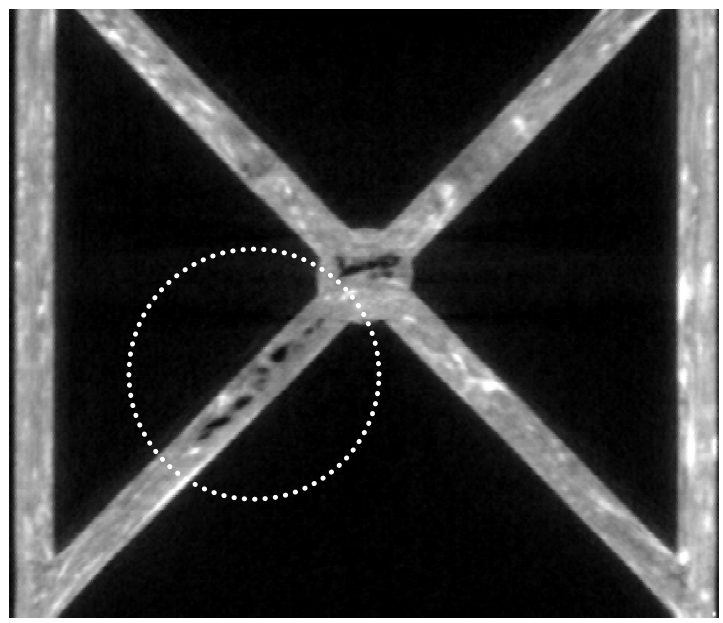

Fig. 6: Reconstructed plane from 3D X-ray micro-tomography showing internal voids (black) in the area where thermographic contrast is obtained. The polymer appears in grey, glass fibres in white

\section{Conclusion}

Sono-thermography has shown its high potential for the sensitive detection of defects positioned relatively deep below the specimen surface in ceramic materials. Forward calculations based on independent information from ultrasound characterisation using an analytical theoretical model for distributed friction heat sources was able to describe the temperature contrast as a function of time. Finally it could be demonstrated that the thermal technique with ultrasonic burst excitation is also able to detect well voids in fibre reinforced thermoplastic polymers occurring due to incorrect production parameters. The combined thermal and non-thermal nondestructive testing approach is very useful to evaluate defect indications appearing in thermographic testing.

\section{REFERENCES}

[1] Z. Ouyang, L.D. Favro, R.L. Thomas, and X. Han: "Theoretical modeling of thermosonic imaging of cracks", Review of quantitative nondestructive evaluation, Vol. 21, ed. by D.O. Thompson and D. E. Chimenti (2002) pp. 577581

[2] J. Rantala, D. Wu, and G. Busse, "Amplitude modulated lock-in vibrothermography for NDE of polymers and composites", in: Research in Nondestructive Evaluation, Vol. 7 (1996) pp. 215-218

[3] H. S. Carslaw and J. C. Jaeger: "Conduction of heat in solids", (Oxford University Press, Oxford 1959) 